Pacific Journal of Mathematics

ON GENERATING SUBGROUPS OF THE MOEBIUS GROUP BY
PAIRS OF INFINITESIMAL TRANSFORMATIONS 


\title{
ON GENERATING SUBGROUPS OF THE MOEBIUS GROUP BY PAIRS OF INFINITESIMAL TRANSFORMATIONS
}

\author{
FrankLin LowenthaL
}

The Moebius group, i.e., the set of all transformations of the form $w=(a z+b) /(c z+d), a, b, c$ and $d$ complex numbers such that $a d-b c \neq 0$, and its connected subgroups have been extensively studied. Its one-parameter subgroups are easily determined; the subgroup generated by a pair of such one-parameter subgroups or by their infinitesimal transformations will be defined in the usual manner. It is found that all except one, to within an inner automorphism, of the connected subgroups of the Moebius group can be generated by an appropriate pair of infinitesimal transformations. Further it is shown that the necessary and sufficient condition that a pair of infinitesimal transformations generate the entire Moebius group is that there is no Hermitian form that is left invariant by both of them. Simple criteria are given to determine whether a given pair of infinitesimal generators satisfy this condition.

The infinitesimal transformations, i.e., elements of the Lie algebra, of the Moebius group (denoted by $H$-Lie algebra- $h$ ) are quadratics: $a z^{2}+2 b z+c, a, b$ and $c$ complex constants. This means that the one-parameter subgroups of $H$ are the solutions of the differential system:

$$
\frac{d w}{d t}=a w^{2}+2 b w+c, \quad w(0, z)=z
$$

i.e., for each $t,-\infty<t<\infty, w(t, z)$ is an element of $H$ and the set of all elements of $H$ that are solutions of (1) form a one-parameter subgroup. It can easily be shown that under the transformations of the Lie algebra induced by inner automorphisms on the group, the discriminant $\left(b^{2}-a c\right)$ of the infinitesimal generator is an absolute invariant. In case the discriminant is real, we can classify the generators as follows: negative discriminant-elliptic; zeroparabolic; positive-hyperbolic.

It is easily seen that $H$ may be identified with the group of all $2 \times 2$ complex matrices having determinant equal to +1 , provided that we identify the matrices $A$ and $-A$, for one may always normalize the transformation $w=(a z+b) /(c z+d)$ with $a d-b c \neq 0$ so as to make $a d-b c$ equal to +1 .

The subgroup generated by a pair of infinitesimal transformations 
is defined to be the smallest topologically closed (with respect to $H$ ) subgroup that contains the one-parameter subgroups generated by each of the two infinitesimal transformations. Thus it consists of all finite products of elements of each of the two one-parameter subgroups together with all nonsingular limits. It turns out that no new transformations are obtained by taking nonsingular limits. This can be seen as follows: the set of all finite products is an arcwise connected subgroup of a Lie group and Yamabe [4] showed that this implies that it is in fact a sub-Lie group. Malcev's theorem [3] asserts that a connected sub-Lie group is closed if and only if it contains the closure of each of its one-parameter subgroups, and it is easily seen that all the one-parameter subgroups of the Moebius group are closed. Therefore it follows that the subgroup of all finite products must itself be closed.

The sub-Lie group generated by a pair of infinitesimal transformations has associated with it a subalgebra of $h$; this is called the generated subalgebra - it is a linear space closed under the bracket operation, which for $h$ is given by:

$$
[\varepsilon(w), \eta(w)]=\eta(w) \frac{d \varepsilon}{d w}-\varepsilon(w) \frac{d \eta}{d w} .
$$

For connected Lie groups it is well known that if the generated subalgebra is the entire Lie algebra, then the generated subgroup is the whole group. Any split semisimple Lie algebra can be generated by two elements [2], and since these conditions are satisfied by $h$, it follows that some pair of infinitesimal transformations generate the whole group $H$; Theorem 1 furnishes a criterion which tells whether a pair of infinitesimal transformations does in fact generate the whole group. Theorem 2 tells which connected subgroups of $H$ can be generated by a pair of infinitesimal transformations. It is of interest to mention that for the real projective group on the real line, i.e., the set of all transformations of the form $y=(a x+b) /(c x+d), a, b, c$ and $d$ all real and $a d-b c \neq 0, a$ pair of infinitesimal transformations will generate the entire connected component of the identity if and only if they have no common root.

The following is a list of all the connected subgroups of $H$ to within an inner automorphism, a result that can be established using the theory of Lie algebras (cf. [1]).

(1) the whole group $H-6$ dimensional;

(2) $w=\alpha z+\beta, \alpha \neq 0, \alpha, \beta$ arbitrary complex numbers-this is the similarity group and is 4 dimensional; $\infty$ is the common fixed point of all transformations of this subgroup;

(3) isometry group of the hyperbolic geometry-all Moebius 
transformations that take the unit circle into itself, interior going into the interior-3 dimensional;

(4) isometry group of the spherical geometry - all linear fractional transformations which under stereographic projection correspond to rotations of the Riemann sphere-3 dimensional;

(5) $w=\alpha z+\beta,|\alpha|=1$-this is a 3 dimensional subgroup of the similarity group which is just the isometries of the Euclidean geometry;

(6) $w=e^{(1+b i) t} z+\hat{o},-\infty<t<\infty, \hat{o}$ arbitrary, $b$ real, fixed-for each $b$ we obtain a 3 dimensional subgroup of the similarity group $(b=0$ yields $w=a z+\delta, a>0)$;

(7) $w=z+\delta-2$ dimensional;

(8) $w=a z+b, a>0, b$ real -2 dimensional;

(9) $w=\lambda z, \lambda \neq 0-2$ dimensional;

(10) the one-parameter subgroups.

The Hermitian form $a z \bar{z}+\bar{b} z+b \bar{z}+c, a$ and $c$ real, is said to be invariant under the Moebius transformation $w=(\alpha z+\beta) /(\gamma z+\delta)$, $\alpha \delta-\beta \gamma \neq 0$ if:

$$
a w \bar{w}+\bar{b} w+b \bar{w}+c=\frac{1}{(\gamma z+\delta)(\overline{\gamma z}+\bar{\delta})}(a z \bar{z}+\bar{b} z+b \bar{z}+c) .
$$

It is easily seen that if one introduces coordinates $z_{1}, z_{2}, w_{1}$, and $w_{2}$ by requiring $w=\left(w_{1}\right) / w_{2}, z=\left(z_{1}\right) / z_{2}$, then the above definition of invariance reduces to the familiar one. A one-parameter subgroup $w=T_{t}(z)$ with infinitesimal generator given by

$$
\frac{d w}{d t}=\dot{w}=\alpha w^{2}+2 \beta w+\gamma
$$

leaves the Hermitian form in (3) invariant if and only if for some real $d$ :

$$
a \dot{w} \bar{w}+a w \overline{\dot{w}}+\bar{b} \dot{w}+b \overline{\dot{w}}=(\alpha z+\overline{\alpha z}+d)(a z \bar{z}+\bar{b} z+b \bar{z}+c)
$$

(left side is evaluated at $t=0$-then $w=z$ ). This reduces to:

$$
\begin{aligned}
& a\left(\alpha z^{2}+2 \beta z+\gamma\right) \bar{z}+a z\left(\overline{\alpha z}^{2}+2 \overline{\beta z}+\bar{\gamma}\right)+\bar{b}\left(\alpha z^{2}+2 \beta z+\gamma\right) \\
& \quad+b\left(\overline{\alpha z}^{2}+2 \overline{\beta z}+\bar{\gamma}\right)=(\alpha z+\overline{\alpha z}+d)(\alpha z \bar{z}+\bar{b} z+b \bar{z}+c) .
\end{aligned}
$$

Given $a, b$ and $c, a$ and $c$ real, this equation imposes conditions on $\alpha, \beta$ and $\gamma$ and conversely.

Any Hermitian form $a z \bar{z}+\bar{b} z+b \bar{z}+c$ is Hermitely congruent to one of the following 3 forms: 

(a) $z \bar{z}+1$
(b) $z \bar{z}-1$
(c) 1

and hence it suffices to study only these forms.

One easily finds from (5) that $z \bar{z}+1$ is left invariant by infinitesimal generators if and only if they are of the form: $(d w / d t)=\alpha w^{2}+2 i b w+\bar{\alpha}$ where $b$ is real-clearly this is an elliptic generator and further the fixed points $z_{1}, z_{2}$ satisfy $z_{1} \bar{z}_{2}=-1 . \quad z \bar{z}-1$ is left invariant by: $(d w / d t)=\alpha w^{2}+2 i b w-\bar{\alpha}$-clearly the discriminant must be real. Finally 1 is left invariant if and only if $\alpha=0$, i.e., $(d w / d t)=2 \beta w+\gamma$.

One observes that every proper connected subgroup of $H$ leaves some Hermitian form invariant; the similarity subgroup (and all its subgroups) leaves 1 invariant; the isometries of the hyperbolic geometry leave $z \bar{z}-1$ invariant and the isometries of the spherical geometry leave $z \bar{z}+1$ invariant. Clearly the whole group $H$ does not leave any Hermitian form invariant.

THEOREM 1. A pair of infinitesimal transformations generate the whole group $H$ if and only if there is no Hermitian form that is left invariant by both of them.

REMARK. If a Hermitian form is left jointly invariant by both generators, then the locus of points where the form is zero can be considered a joint orbit of the generators-this is a real locus except in the positive definite case and to within an inner automorphism the real joint orbit is always either the unit circle or the point at infinity.

CoRollary 1. If an infinitesimal transformation has discriminant with nonzero imaginary part, then the subgroup generated by it and any other infinitesimal generator equals $H$ if and only if they do not have a common fixed point.

Proof. The necessity of the condition is obvious; to prove the sufficiency we note that if a proper subgroup is generated, then there is a Hermitian form left invariant. Since one generator has discriminant with nonzero imaginary part, it cannot leave the forms $z \bar{z}+1$ or $z \bar{z}-1$ invariant, and hence the Hermitian form left jointly invariant may be assumed to be 1 so that $\infty$ must be a common fixed point.

A parabolic generator is determined by knowledge of its fixed point and fixed line element; the roots of a parabolic generator are defined as any two distinct points on this line element. If $z_{0}, z_{1}$ are the fixed points of one infinitesimal transformation, $w_{0}, w_{1}$ of another, 
then the cross-ratio of these fixed points is the expression: $\left(z_{0}, z_{1} ; w_{0}, w_{1}\right)$.

COROLlARY 2. Let $\varepsilon$ and $\eta$ be elements of $h$ with real discriminants and no common roots. Then if exactly one of them is elliptic, the generated subgroup is equal to the whole group $H$ if and only if the absolute value of the cross-ratio of the fixed points is not equal to unity. In all other cases $H$ is generated if and only if this crossratio is not real.

Proof. Suppose first that neither generator is elliptic and that they have no common root but generate a proper subgroup of $H$. Then clearly the Hermitian form $z \bar{z}-1$ or equivalently $i z-i \bar{z}$ may be assumed to be left invariant by both generators, i.e., the real axis may be assumed to be a joint orbit and hence all fixed points lie on a line so that the cross-ratio is real.

If one generator is elliptic, the other not and there is no common fixed point but a proper subgroup of $H$ is generated, then we may again assume that the real axis is a joint orbit. Thus the roots of the elliptic generator are complex conjugates and those of the nonelliptic generator are real. But $|(a, b ; z, \bar{z})|=1$ provided $a$ and $b$ are real.

If both generators are elliptic without common fixed points but generate a proper subgroup of $H$, then either $z \bar{z}+1$ or $i z-i \bar{z}$ may be assumed to be left invariant. In the former case one knows that the roots of each generator satisfy $z_{1} \bar{z}_{2}=-1$ and one verifies that: $(z,(-1) / z ; w,(-1) / w)$ is a negative number $(z \neq w)$. Thus the fixed points lie on a circle and interlace. In case the real axis is a joint orbit, the roots of each generator are complex conjugates and one verifies that $(z, \bar{z} ; w, \bar{w})$ is a positive number $(z \neq w)$. Thus the fixed points are on a circle and separate.

The steps in each of the arguments above are reversible. For example, if two elliptic generators with no common roots have a negative cross-ratio for their roots, one can find a Moebius transformation that takes them into $0, \infty$ and $a,-(1) / \bar{\alpha}$ respectively for some $a$ ( $a$ not unique). The new generators will then both leave the Hermitian form $z \bar{z}+1$ invariant.

Finally one is interested in what proper subgroups of $H$ can be generated by a pair of infinitesimal transformations.

THEOREm 2. All the connected subgroups of the Moebius group can be generated by an appropriate pair of infinitesimal transformations, with one exception. The subgroup of $H ; w=a z+\beta$, $a>0, \beta$ complex, a three dimensional subgroup, cannot be generated by any pair of infinitesimal generators. 
Proof. One should refer to the list of connected subgroups of $H$ by number.

(1) The whole group $H$-already considered.

(2) The similarity group-let $\varepsilon=i w, \eta=w+1$.

The subgroup generated by $\varepsilon$ and $\eta$ is just the similarity group, for one observes that $\infty$ is a common fixed point of $\varepsilon$ and $\eta$ and a simple calculation shows that the generated subgroup is an least four dimensional.

(3) Isometry group of the hyperbolic geometry-take any two elliptic generators whose roots lie on a circle and separate.

(4) Isometry group of the spherical geometry-take any two elliptic generators whose roots lie on a circle and interlace.

(5) Isometry group of the Euclidean geometry-let $\varepsilon=i w, \eta=$ $i w+1$. One observes that the generated subgroup is at most a three dimensional subgroup of the similarity group and it is easily seen that $\varepsilon, \eta$ and $[\varepsilon, \eta]$ are linearly independent over the reals.

(6) $w=e^{(1+b i) t} z+\delta,-\infty<t<\infty, \delta$ arbitrary, $b$ fixed-it is assumed that $b$ is not zero. Let $\varepsilon=(1+b i) w, \eta=(1+b i) w+1$. Verification is completely similar to the preceding case.

(7) Translation group-let $\varepsilon=1, \eta=i$.

(8) $w=a z+b, a>0, b$ real-let $\varepsilon=w, \eta=1$.

(9) $w=\lambda z, \lambda \neq 0$-let $\varepsilon=w, \eta=i w$.

It remains to prove that $w=a z+\beta, a>0, \beta$ complex is not generated by any pair of infinitesimal transformations. One observes that the subalgebra of $h$ associated with this subgroup has a basis $\varepsilon_{1}=w, \varepsilon_{2}=1, \varepsilon_{3}=i$; one need only show that for any pair $\varepsilon$ and $\eta$ of the form:

$$
\varepsilon=b_{1} w+b_{2}+b_{3} i, \quad \eta=c_{1} w+c_{2}+c_{3} i
$$

(all coefficients real), there exist real constants $r$ and $s$ such that

$$
[\varepsilon, \eta]=r \varepsilon+s \eta \text {. }
$$

But one finds that in fact:

$$
[\varepsilon, \eta]=b_{1} \eta-c_{1} \varepsilon .
$$

This completes the proof of Theorem 2 .

The author wishes to acknowledge his debt to the late Professor Charles Loewner. 


\section{BIBLIOGRAPHY}

1. L. Ahlfors, A Course in Automorphic Functions, given at Harvard University, 1960-61.

2. Nathan Jacobson, Lie Algebras, Interscience Publishers, 1962.

3. A. Malcev, On the theory of Lie groups in the large, Mat. Sbornik 16 (1945), 163189.

4. Hidehiko Yamabe, On an arcwise connected subgroup of a Lie Group, Osaka Mathematical Journal 2 (1950), 13-14.

Received September 14, 1965, and in revised form December 1, 1966. The main theorem in this paper is a revision of a result included in this author's doctoral dissertation. This research was partially supported by a summer research grant of the University of Oregon. 



\section{PACIFIC JOURNAL OF MATHEMATICS}

\section{EDITORS}

\author{
H. ROYDEN \\ Stanford University \\ Stanford, California \\ R. R. Phelps \\ University of Washington \\ Seattle, Washington 98105
}

J. DUGUNDJI

Department of Mathematics

University of Southern California

Los Angeles, California 90007

RICHARD ARENS

University of California

Los Angeles, California 90024

\section{ASSOCIATE EDITORS}
E. F. BECKENBACH
B. H. NeumanN
F. WOLF
K. YosidA

\section{SUPPORTING INSTITUTIONS}

UNIVERSITY OF BRITISH COLUMBIA

CALIFORNIA INSTITUTE OF TECHNOLOGY

UNIVERSITY OF CALIFORNIA

MONTANA STATE UNIVERSITY

UNIVERSITY OF NEVADA

NEW MEXICO STATE UNIVERSITY

OREGON STATE UNIVERSITY

UNIVERSITY OF OREGON

OSAKA UNIVERSITY

UNIVERSITY OF SOUTHERN CALIFORNIA
STANFORD UNIVERSITY

UNIVERSITY OF TOKYO

UNIVERSITY OF UTAH

WASHINGTON STATE UNIVERSITY

UNIVERSITY OF WASHINGTON

AMERICAN MATHEMATICAL SOCIETY CHEVRON RESEARCH CORPORATION TRW SYSTEMS

NAVAL WEAPONS CENTER

Printed in Japan by International Academic Printing Co., Ltd., Tokyo, Japan 


\section{Pacific Journal of Mathematics}

\section{Vol. 26, No. $1 \quad$ November, 1968}

Efraim Pacillas Armendariz, Closure properties in radical theory......... 1

Friedrich-Wilhelm Bauer, Postnikov-decompositions of functors .......... 9

Thomas $\mathrm{Ru}-$ Wen Chow, The equivalence of group invariant positive definite

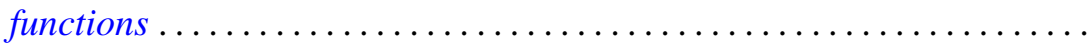

Thomas Allan Cootz, A maximum principle and geometric properties of

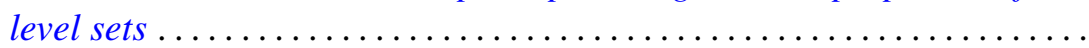

Rodolfo DeSapio, Almost diffeomorphisms of manifolds ............ 47

R. L. Duncan, Some continuity properties of the Schnirelmann density......

Ralph Jasper Faudree, Jr., Automorphism groups of finite subgroups of

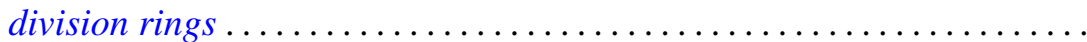

Thomas Alastair Gillespie, An invariant subspace theorem of $J$.

Feldman.........................................

George Isaac Glauberman and John Griggs Thompson, Weakly closed direct factors of Sylow subgroups .............................

Hiroshi Haruki, On inequalities generalizing a Pythagorean functional equation and Jensen's functional equation .....................

David Wilson Henderson, D-dimension. I. A new transfinite dimension.....

David Wilson Henderson, D-dimension. II. Separable spaces and

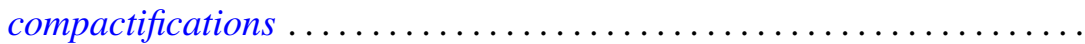

Julien O. Hennefeld, A note on the Arens products ............... 115

Richard Vincent Kadison, Strong continuity of operator functions ...

J. G. Kalbfleisch and Ralph Gordon Stanton, Maximal and minimal coverings of $(k-1)$-tuples by $k$-tuples.

Franklin Lowenthal, On generating subgroups of the Moebius group by pairs of infinitesimal transformations...

Michael Barry Marcus, Gaussian processes with stationary increments possessing discontinuous sample paths . .

Zalman Rubinstein, On a problem of Ilyeff ...

Bernard Russo, Unimodular contractions in Hilbert space. ...

David Lee Skoug, Generalized Ilstow and Feynman integrals...

William Charles Waterhouse, Dual groups of vector spaces . 